\title{
Pregnancy-associated breast cancer: tumor features and obstetrical outcomes in a single institution retrospective study
}

\begin{abstract}
Purpose: Pregnancy-associated breast cancer (PABC) is one of the most common malignancies during pregnancy. Our objective is to analyze the maternal and foetal outcomes in women with PABC.

Methods: We conducted a retrospective study of 14 patients diagnosed and treated for PABC at our institution between 1998 and 2012, in order to analyze tumor biological features and to determine treatment's safety.

Results: Median maternal age at diagnosis was 33.64 years. 1 patient was classified as having clinical stage I disease, 10 as stage II, 1 as stage III, 2 as stage IV. Pregnancy was discontinued in 2 patients, for patients' choice. Treatment was started during pregnancy in all cases. 5 women underwent surgery during pregnancy, 2 in the first trimester, 2 in the second trimester and 1 in the third trimester. Six women received chemotherapy during pregnancy. The median gestational age at chemotherapy start was $23.33+6.52$ sd weeks. The median gestational age at delivery was $35.2+8.67$ weeks. In 2 cases premature delivery followed spontaneous preterm labour; in 6 cases early delivery was planned to optimize treatment. Median weight was $2381.67 \pm 632.60 \mathrm{sd} \mathrm{kg}$. APGAR Score was 7.56 $\pm 2.60 \mathrm{sd}$ at 1 minute and $8.33 \pm 1.31 \mathrm{sd}$ at 5 minutes. Among the six foetuses exposed to chemotherapy in utero, no miscarriages or foetal malformations were reported. At a median follow-up of $88,21+67,06$ sd months, 10 patients were alive and free of disease, 1 was alive with disease, and 3 patients had died because of breast cancer.

Conclusion: Chemotherapy during the second and the third trimester is safe, and surgery can safely be performed in any trimester. Delivery should not be induced before the 37 th week, as iatrogenic preterm birth is the principal responsible of negative long-term outcomes.
\end{abstract}

Volume 7 Issue I - 2017

\author{
Elisa Picardo, ${ }^{2}$ M Porpiglia,' M Mitidieri, ${ }^{3} \mathrm{~F}$ \\ Borella, ${ }^{2}$ R Arisio, ${ }^{3}$ C Benedetto ${ }^{2}$ \\ 'A.O.U. Citta della Salute e della Scienza, Gynecology and \\ Obstetrics I, SantAnna Hospital, Italy \\ ${ }^{2}$ Gynecology and Obstetrics I, Department of Surgical Sciences, \\ University of Torino, Italy \\ ${ }^{3}$ Department of Surgical Sciences, University of Torino, Italy \\ ${ }^{4}$ A.O.U. Citta della Salute e della Scienza, SantAnna Hospital, \\ Anatomia ed istologia patologica della donna, del bambino e dei \\ tumori rari, Italy
}

Correspondence: Elisa Picardo, University of Torino, via ventimiglia 3,Torino, Italy, Email elisa.picardo@gmail.com

Received: September 14, 2016 | Published: May II, 2017

\section{Introduction}

Pregnancy-associated breast cancer (PABC) is defined as the breast cancer, which is diagnosed during pregnancy or within 1 year of delivery. Most experts believe that the majority of these breast cancers have been present for 2 years before clinical detection. Hence, breast cancers diagnosed up to 1 year postpartum were most likely present during pregnancy. ${ }^{1}$

Breast cancer is the most common malignancy occurring during pregnancy. It has been estimated that approximately 1 of 3000 deliveries occurring in women with breast cancer. The diagnosis of breast cancer in pregnancy is expected to become more frequent since there is an increasing trend for women to delay childbearing. ${ }^{2-4}$ However the breast cancer is a rare event in young women under 40 years old affecting less than $10 \%$ of patients with breast cancer, but in developed countries is the most common cause of death in 30 years old women and also the incidence is greater as compared to other cancers. $^{5}$

The tumours diagnosed at young age are more aggressive than those diagnosed at older age and because pregnant patients are young, breast cancer during pregnancy is more aggressive and often develops at a later stage than women not in pregnancy.

Conflicting data exist from the larger published series of PABC regarding the impact of this diagnosis on tumor features and outcomes; further, the commonly limited experience in treating this clinical setting makes it difficult for clinicians to know how best to treat pregnant women with breast cancer. Therefore, there is a need to optimize the treatment of the mother, but at the same time to minimize the risks to the foetus, trying to avoid delayed or under-treatment and inopportune premature delivery and termination of the pregnancy. ${ }^{2-4,6}$

We conducted a retrospective study of patients diagnosed with PABC at our institution, in order to deal the tumor biological features and to determine whether treatment for breast cancer during pregnancy is safe for both mother and foetus, discussing the available data, and providing some guidance as to appropriate strategies.

\section{Material and methods}

We evaluated all patients diagnosed with PABC at the Department of Obstetrics and Gynaecology, University of Turin, and treated at the same institution between 1998 and 2012. Patients with breast cancer diagnosed after delivery were not included in this cohort.

Details about cancer diagnosis, tumor characteristics (size, stage including lymph node status, hormone receptor status and HER2 protein expression), and local and systemic treatment (type of surgery, chemotherapy regimen) were obtained in all individual cases. Maternal and neonatal outcomes were reported, and their follow up is ongoing. 


\section{Results}

This cohort included 14 patients diagnosed with PABC. Median maternal age at diagnosis was 33.64 years old (age range 25 to 40 years old).

Of these 14 women, 3 of them were diagnosed in the first trimester, 4 in the second trimester, and 7 patients in the third trimester, with a median gestational age at diagnosis of $23.31 \pm 10.4$ SD weeks (age range 8 to 39 weeks). 13 patients self-discovered the tumours as palpable masses, and one patient presented with a diffuse erythema (inflammatory carcinoma). At presentation, considering TNM classification system of the American Joint Committee on Cancer, 1 patient was classified as having clinical stage I disease, 10 were classified as having stage II disease, 1 was classified as having stage III disease, 2 were classified as having stage IV disease. All patients received systemic chemotherapy. Of these, 6 were offered chemotherapy in their second and third trimester with anthracyclinebased treatment; one of these received paclitaxel during pregnancy after completing anthracycline four corses. Baseline characteristics of patients, and details about women treated with chemotherapy during pregnancy are shown in Table $1 \& 2$, respectively.

Table I Baseline characteristics

\begin{tabular}{lll}
\hline Baseline characteristics & & \\
\hline \multirow{2}{*}{ Maternal Age at Diagnosis } & Median Age & 33,64 \\
GA (W) at diagnosis & SD & 4,27 \\
& First Trimester & 4 \\
& Second Trimester & 4 \\
Tumor Stage & Third Trimester & 6 \\
& 1 & 1 \\
& 2 & 10 \\
Nodal Status & 3 & 1 \\
& 4 & 2 \\
Istological Type & X & 1 \\
& Negative & 4 \\
Grading & Positive & 9 \\
& Ductal & 11 \\
ER/Pgr status & Lobular & 0 \\
& Other & 3 \\
HER & 1 & 0 \\
& 2 & 3 \\
& 3 & 11 \\
& Negative for both ER or Pgr & 9 \\
& Positive for ER, Pgr, or both & 5 \\
& 0 & 7 \\
& I & 6 \\
\end{tabular}

Table 2 Series of women $(n=6)$ with breast cancer receiving chemotherapy during pregnancy

\begin{tabular}{|c|c|c|c|c|c|c|c|c|c|c|c|}
\hline Patients & $\begin{array}{l}\text { Age at } \\
\text { Diagnosis }(Y)\end{array}$ & $\begin{array}{l}\text { Gestational } \\
\text { Age at } \\
\text { Diagnosis (W) }\end{array}$ & $\begin{array}{l}\text { Gestational } \\
\text { Age at Starting } \\
\text { )Chemotherapy (W) }\end{array}$ & $\begin{array}{l}\text { Cycles } \\
\text { (Numbers) }\end{array}$ & Delivery & $\begin{array}{l}\text { Gestational } \\
\text { Age at } \\
\text { Delivery (W) }\end{array}$ & Sex & PESO & $\begin{array}{l}\text { APGAR } \\
\text { I }\end{array}$ & $\begin{array}{l}\text { APGAR } \\
5\end{array}$ & Abnormalities \\
\hline I & 31 & 16 & 18 & 6 & PS & 37 & M & 1970 & 9 & 9 & 0 \\
\hline 2 & 35 & 27 & 29 & 2 & PS & 35 & M & 2700 & 9 & 9 & 0 \\
\hline 3 & 29 & 28 & 28 & 2 & $\mathrm{TC}$ & 36,2 & M & 2660 & 7 & 8 & 0 \\
\hline 4 & 28 & 32 & 32 & 2 & PS & 36,4 & $\mathrm{~F}$ & 2660 & 8 & 8 & 0 \\
\hline 5 & 32 & 15 & 15 & 5 & $\mathrm{TC}$ & 31 & $\mathrm{~F}$ & 1270 & 7 & 9 & 0 \\
\hline 6 & 32 & 13 & 18 & 5 & $\mathrm{TC}$ & 35,6 & $\mathrm{~F}$ & 2850 & 8 & 9 & Mosaicism \\
\hline
\end{tabular}

11 patients underwent breast-conserving surgery, 3 patients had mastectomies. Axillary lymph node clearance was performed in 13 patients. 5 women underwent surgery during pregnancy, 2 in the first trimester, 2 in the second trimester and 1 in the first trimester. None of the patients underwent immediate reconstruction 6 women received chemotherapy in the adjuvant setting, 7 women in the neoadjuvant setting, while 1 was treated for metastatic breast cancer at presentation. In pregnant patients who required adjuvant radiotherapy, this was postponed until delivery. 6 women received chemotherapy during their pregnancy, with a median of $3.67+1.70 \mathrm{ds}$ cycles (range 2 to 6 ).

The median gestational age at chemotherapy initiation was $23.33+6.52 \mathrm{ds}$ weeks (range 15-32 weeks). 1 patient received epirubicina (E) $120 \mathrm{mg} / \mathrm{mq}$ every 3 weeks. In 5 patients chemotherapy consisted of $75 \mathrm{mg} / \mathrm{m} 2$ epirubicin plus cyclophosphamide $600 \mathrm{mg} / \mathrm{m} 2$
(EC) intravenously in a single dose every 21 days. One of this patients received 2 more cycles of Paclitaxel (P) 1

$75 \mathrm{mg} / \mathrm{mq}$, after 4 cycles of antracycline. The use of growth factor support was reported in none of the patients. Lattation was suppressed in all patients.

\section{Tumor pathological features}

Invasive ductal carcinoma was the histological subtype in 11 patients $(78.6 \%)$. The invasive carcinoma in $3(21.43 \%)$ of the patients was moderately differentiated, and the other 11 of patients had poorly differentiated invasive carcinomas. Ductal carcinoma in situ (DCIS) was associated in $6(42.86 \%)$ of the tumor specimens, while lymphovascular invasion was present in $8(57.14 \%)$ cases. Lymph 
node status was positive in $9(64.28 \%)$ patients. About recettorial expression, ER alone positivity was identified in 4 of the 14 patients (28.57\%), while only 4 patients were positive for both ER and PR. About HER2 status, 6 of the 14 patients $(42.86 \%, 1$ data missing) revealed $3+$ positive membranous staining.

\section{Maternal and neonatal outcomes}

The median gestational age at delivery was $35.2 \pm 8.67$ weeks (range 31 to 39). In 2 cases premature delivery occurred as a result of spontaneous onset of preterm labour; however, in 6 cases early delivery was planned in order to optimize the timing of delivery relative to chemotherapy or further required treatment. Birth weights were available in all babies, with a median weight of $2381.67 \pm$ $632.60 \mathrm{sd} \mathrm{kg}$ (range 1270-3340 kg). The babies had APGAR Score $7.56 \pm 2.60 \mathrm{sd}$ and $8.33 \pm 1.31 \mathrm{sd}$ (at 1 and $5 \mathrm{~min}$, respectively).

In the cohort of six fetuses exposed to chemotherapy in utero, no miscarriages or fetal malformations were reported. The Apgar score in all children born to patients who received antepartum chemotherapy was normal suggesting efficacy and no teratogenic effect of the chemotherapy administered. Complications at birth included respiratory distress syndrome in one neonate iatrogencally delivered at 33 weeks with cesarian section. Only a single neonate was diagnosed with a congenital mosaicism. One patient had distant metastasis at the time of initial presentation (bones), and metastasis subsequently developed in other 4 patients. The first site of subsequent metastasis included lung (1 patient), and nonaxillary lymph nodes (3 patients). At a median follow-up duration of $88,21+67,06 \mathrm{ds}$ months (range 7,80-1234,97months), among the 14 patients: $71.4 \%$ of them $(n=10)$ were alive and free of disease, $7.14 \%$ of them $(n=1)$ were still alive with disease. Of the 3 patients $(21.43 \%$ ) confirmed to have died, all died of metastatic breast cancer.

\section{Discussion}

\section{Diagnosis and Staging}

PABC is often diagnosed at an advanced stage. Several factors may contribute to this. First, it is difficult to evaluate neoplasms in the pregnant female breast: it is due to the engorgement secondary to hormonal changes. Moreover obstetricians frequently direct their attention to the developing fetus rather than perform a comprehensive physical examination. These factors often contribute to a delay in diagnosis and then in advanced disease presentation.

In our series, the primary tumors diameter ranged from 12 to $100 \mathrm{~mm}$ (median $37.21 \pm 24.87 \mathrm{~cm}$ ). 10 patients presented with a solitary tumor mass, 3 patients had multifocal disease, 1 patient had inflammatory carcinoma. Only 1 patient was classified as having TNM clinical stage I disease at presentation.

Breast ultrasonography is the first diagnostic tools assess a breast mass in a pregnant woman. ${ }^{7}$ If the ultrasound results appear suspicious a core biopsy, and a subsequent mammography can be performed. Taken into account that the increased density of the breast during pregnancy may reduce the sensitivity of mammography. Metastatic investigations for breast cancer during pregnancy, if necessary, include chest radiograph, liver ultrasonography, and a non-contrast skeletal MRI. In fact, there have been studies in animal models showing that gadolinium crossing the placenta is associated with fetal abnormalities. $^{8}$

\section{Pathological changes}

From histopathological point of view tumor features during pregnancy seem to be determined mostly by the age:according to the most recent literature data, in our study we confirmed that the pathological findings of breast cancer are similar to those in nopregnant women who are younger than 35 years. Despite these features previous data from literature. ${ }^{9-12}$ Shows that most pregnant patients are diagnosed with infiltrating ductal adenocarcinomas (71$100 \%$ ), which are often associated with aggressive behaviour, high incidence of grade 3 tumours (40-95\%), lymphovascular invasion, high rate of proliferation index, and a high rate of oestrogen receptor negativity. ${ }^{13}$ Gestational breast cancer is associated with larger tumours and a higher incidence of nodal involvement (53-71\%) than in non-pregnant patients. About HER2 expression the studies are inconclusive, although data from a recent study on more than 300 patients showed HER 2 positivity in $42 \%$ of them which is almost the same $(39 \%)$ as recorded in non-pregnant patients with breast cancer younger than 35 years. ${ }^{14,15}$

\section{Treatment}

Counselling is of utmost importance because of the complexity of the issue. A multidisciplinary team with all involved specialties should assess the medical, ethical, and psychological issues. The proposed treatment should adhere to the standard for non-pregnant patients. Preterm delivery or unnecessary delay in diagnosis or treatment to the post-partum period should be avoided. Because preterm birth is strongly associated with adverse events, it can be prevented by cancer treatment during pregnancy, and a full-term delivery seems to be a paramount importance. ${ }^{14}$.

\section{Pregnancy termination}

In our series, pregnancy was discontinued in 2 patients, because of their own decision. In both cases, women and their partners have been informed that termination of pregnancy does not seem to improve maternal outcome, and that several reports suggest a trend toward shorter survival. ${ }^{4}$ Only when chemotherapy is required during the first 12 weeks of amenorrhea (eg, metastatic disease at presentation) pregnancy termination should be considered, due to the significant risk of fetal malformation.

\section{Surgery}

In our cohort, four women underwent surgery during pregnancy, two in the first trimester and two in the second trimester (Table 3 ). In general, surgery can be performed safely during any stage of pregnancy and most anaesthetic agents seem to be safe for the fetus. ${ }^{15-17}$ ] Fetal heart-rate monitoring during surgery should be used to detect fetal distress. ${ }^{8}$ The choice of breast cancer surgery during pregnancy should follow the same guidelines as for non-pregnant women. Clinicians can safely use sentinel lymph-node staging during pregnancy. ${ }^{18-20}$ The estimated absorbed doses after injection of 92.5 $\mathrm{MBq}$ of technetium $99 \mathrm{~m}$-labelled sulphur colloid is safe for the fetus, while the use of blue dye should be avoided because is associated with a risk of an anaphylactic maternal reaction.$^{18,21}$

Radiation during pregnancy carries a risk of fetal exposure to the radiation field. Because surgery and the initiation of systemic therapies are often completed prior to the initiation of radiation, radthiiation therapy can be delayed until after delivery. If breast reconstruction is considered, a prosthetic implants is possible but in view of physiological changes, autologous reconstruction should be delayed until after delivery. ${ }^{22}$

\section{Cytotoxic treatment}

For breast cancer in pregnancy, the decision to administer chemotherapy should follow the same guidelines as in non-pregnant patients. In this analysis, the safety of administering chemotherapy 
during the second and the third trimester of pregnancy was evaluated in a small number of patients $(n=6)$. All of whom received anthracyclinebased treatment, and one woman received two additional cycles of paclitaxel, even if most guidelines for breast cancer do not recommend taxanes during pregnancy. However, the reported complications of the infants if taxanes were given during pregnancy did not differ from those of other cytotoxic agents.

Table 3 Breast cancer TNM and type of surgery

\begin{tabular}{|c|c|c|c|c|c|c|c|}
\hline Patients & $\mathbf{T}$ & $\mathbf{N}$ & $\mathbf{M}$ & Surgery & Limphonodes surgery & Gestational age at surgery $(y)$ & Status \\
\hline l & 2 & la & 0 & QUA & $\mathrm{DA}$ & 19 & died \\
\hline 2 & 2 & I & 0 & QUA & DA & Post & ned \\
\hline 3 & 2 & I & 0 & QUA & DA & Post & ned \\
\hline 4 & 2 & I & I & QUA & DA & Post & Bone metastases \\
\hline 5 & Ic & 0 & 0 & QUA & DA & 25 & ned \\
\hline 6 & $4 d$ & $3 a$ & 0 & MA & DA & POST & died \\
\hline 7 & 2 & 0 & 0 & QUA & BLS & 39 & ned \\
\hline 8 & 2 & $2 a$ & 0 & QUA & DA & POST & died \\
\hline 9 & 2 & 0 & 0 & QUA & DA & POST & ned \\
\hline 10 & 2 & 0 & 0 & QUA & DA & 13 & Loco regional recurrence \\
\hline II & 3 & I & 0 & QUA & BLS & Post & ned \\
\hline 12 & 2 & 1 & $x$ & QUA & DA & Post & ned \\
\hline 13 & $4 b$ & $x$ & 0 & MA & BLS & Post & ned \\
\hline 14 & 2 & $2 a$ & 0 & MA & DA & 13 & ned \\
\hline
\end{tabular}

The effect of the administration of cytotoxic treatment on a pregnancy varies and depends on the gestational stage during exposure. Reports of fetal malformations have been in the range of $14-19 \%$ when chemotherapy has been given in the period of organogenesis (10 days to 8 weeks after conception). Several data confirm an important barrier function for the placenta: for fetal protection, chemotherapy is contraindicated until 10 weeks' gestation. With a safety period of 4 weeks, chemotherapy can start from 14 weeks' gestation. Cytotoxic treatment in the second and third trimester is associated with a reported fetal malformation rate of about $3.0 \%$, which is not higher than that descripted in the general population, although researches have reported growth restriction, intrauterine and neonatal death, prematurity, and haemopoietic suppression. ${ }^{23}$ In our series among the six fetuses exposed to chemotherapy we have not observed any complication, and only one neonate was born with mosaicism. On the basis of theoretical assumptions, neurodevelopment problems, carcinogenesis, sterility, and genetic defects are possible, but data on long-term outcomes after prenatal exposure to chemotherapy are poo. $[24,25$ a study of 84 children who were born to mothers who received chemotherapy during pregnancy for haematological malignancies with an average follow-up of 19 years did not show any abnormalities. ${ }^{26}$

Chemotherapy can be adjuvant or neoadjuvant, and clinicians can use standard regimens such as fluorouracil and epirubicin or doxorubicin plus cyclophosphamide, or epirubicin or doxorubicin plus cyclophosphamide and taxanes (paclitaxel weekly to every 3 weeks or docetaxel every 3 weeks). There is lack of data on dosedense chemotherapeutic regimens during pregnancy. The dose should be calculated on the basis of actual weight and body surface area. ${ }^{27-30}$

The major causes of undesiderable fetal outcome appear to be derived from premature delivery, rather than from any direct effect of the chemotherapy. We reported one newborn with respiratory distress syndrome after caesarean delivery at 33 weeks but the woman didn't receive chemotherapy during pregnancy. Therefore, iatrogenic preterm delivery should be avoided as much as possible, and no chemotherapy doses should be given after 35 weeks of gestation to avoid delivery of leukopenic infant. ${ }^{31}$

Use of trastuzumab needs to be further investigated. In our series two women received trastuzumab without significant toxicity for the patients and the foetuses ${ }^{32-34}$ Tamoxifen is not recommended because of birth defects associated with its exposure.

\section{Prognosis}

Conflicting data exist from the larger published series of PABC regarding the maternal prognosis. Recent data (Rodriguez 2008) concluded that women with PABC presented with more advanced disease, large tumours, and an increased percentage of nodal involvement. It's unclear whether this is due to less aggressive therapy secondary to concern for fetal effects, a later stage at diagnosis due to the difficulties of diagnosing $\mathrm{PABC}$, or physiologic changes in pregnancy that contribute to worse outcomes, or a combination of these factors. Prospective observational studies ad long-term follow up could provide a better understanding of the problem..$^{35}$

\section{Prenatal care}

No guidelines have been issued for obstetricians to monitor pregnant patients treated for breast cancer, therefore these women should be followed up and treated in a high-risk obstetric patients unit. Before staging examinations or oncological treatment is started, fetal structural development and growth should be assessed to exclude pre-existing anomalies. ${ }^{13}$ Since in a series of 215 patients, the risk of preterm labour and growth restriction were increased, perinatologists should pay special attention to these adverse events [3]. When anthracyclines are used, special consideration should be given when maternal conditions involving the cardiovascular system are apparent (eg, pre-eclampsia). The aim of a term delivery $(>37$ weeks' gestation) is important since prematurity is related to more paediatric events. When breast cancer in pregnancy is diagnosed in the third trimester and when only one cycle of chemotherapy is needed before fetal maturity, delivery at 35 weeks and postnatal start of chemotherapy can be considered. ${ }^{27}$ This decision is a balance between the risks of late prematurity and the poorly documented long-term outcome after chemotherapy exposure late in pregnancy. A three week interval should be left between the last cycle of chemotherapy and the delivery to avoid problems associated with haemopoietic suppression (bleeding, infection, anaemia) in the mother and baby, and to avoid drug accumulation in the fetus. ${ }^{6,13}$ 
No cases of fetal metastases are described, but pathological evaluation of the placenta should be recommended, because of the isolated reports of placental metastases. Postpartum oncological treatment, including chemotherapy and radiotherapy, can be restarted immediately after a vaginal delivery. After an uncomplicated caesarean section, an interval of a week is recommended before oncological treatment is continued. Breastfeeding in the first weeks after chemotherapy is not recommended.

In our series children exposed to chemotherapy in utero have shown no side effects. The largest study with a follow up of five years looked at 84 children exposed to chemotherapy in utero and followed for more than 18 years, did not observe any kind of abnormalities or malignancies. $^{26}$

\section{Conclusion}

The results of our study show that efficient treatment for breast cancer during pregnancy is possible. In fact, we confirmed that administering chemotherapy during the second and the third trimester is safe, and that even surgery can safely be performed on pregnant women in any trimester. A multidisciplinary discussion should determine the therapeutic strategy: cancer treatment should adhere as much as possible to the standardised protocols for non-pregnant women. Since management is critically influenced by the stage of pregnancy, such patients require a tailored approach to treatment. If possible, delivery should not be induced before the 37 th week. In fact, iatrogenic preterm birth is principal responsible of negative long-term outcomes, irrespective of exposure to chemotherapy, but it can be prevented by cancer treatment during pregnancy. Maternal prognosis needs to be defined. Data concerning the long-term risks of systemic anticancer treatment are limited: owing to the lack of randomized trials, long-term follow-up are needed to provide further information.

\section{Acknowledgments}

None.

\section{Conflicts of interest}

None.

\section{References}

1. Robert A Aronowitz. Unnatural history: breast cancer and American society. N Engl J Med. 2007;359:1635-1636.

2. Stensheim H, Møller B, van Dijk T, et al. Cause-specific survival for women diagnosed with cancer during pregnancy or lactation: a registrybased cohort study. J Clin Oncol. 2009;27(1):45-51.

3. Van Calsteren K, Heyns L, De Smet F, et al. Cancer during pregnancy: an analysis of 215 patients emphasizing the obstetrical and the neonatal outcomes. J Clin Oncol. 2010;28(4):683-689.

4. Cardonick E, Dougherty R, Grana G, et al. Breast cancer during pregnancy: maternal and fetal outcomes. Cancer J. 2010;16(1):76-82.

5. Gentilini O. Breast cancer during pregnancy: epidemiology, surgical treatment and staging. Recent Results Cancer Res. 2008;178:39-44.

6. Wohlfahrt J, Olsen JH, Melby M. Breast cancer risk after childbirth in young women with family history (Denmark). Cancer Causes Control. 2002;13(2):169-174.

7. Yang WT, Dryden MJ, Gwyn K, et al. Imaging of breast cancer diagnosed and treated with chemotherapy during pregnancy. Radiology. 2006;239(1):52-60.
8. Amant F, Deckers S, Van Calsteren K, et al. Breast cancer in pregnancy: recommendations of an international consensus meeting. Eur J Cancer. 2010;46(18):3158-3168

9. Ishida T, Yokoe T, Kasumi F, et al. Clinicopathologic characteristics and prognosis of breast cancer patients associated with pregnancy and lactation: analysis of case-control study in Japan. Jpn J Cancer Res. 1992;83(11):1143-1149.

10. Loibl S, Amant F, Kaufmann M, et al. 313 patients with breast cancer during pregnancy-a prospective and retrospective registry (GBG-20/ BIG02-03). San Antonio Breast Cancer Symposium; San Antonio, Texas, USA. 2010.

11. Elledge RM, Ciocca DR, Langone G, et al. Estrogen receptor, progesterone receptor, and HER-2/neu protein in breast cancers from pregnant patients. Cancer. 1993;71(8):2499-2506.

12. Loibl S, von Minckwitz G, Gwyn K, et al. Breast carcinoma during pregnancy. International recommendations from an expert meeting. Cancer. 2006;106(2):237-246.

13. Ni Mhuireachtaigh $\mathrm{R}, \mathrm{O}$ Gorman DA. Anesthesia in pregnant patients for nonobstetric surgery. J Clin Anesth 2006;18(1):60-66.

14. Moran BJ, Yano H, Al Zahir N, et al. Conflicting priorities in surgical intervention for cancer in pregnancy. Lancet Oncol. 2007;8(6):536-544.

15. Cohen Kerem R, Railton C, Oren D, et al. Pregnancy outcome following non-obstetric surgical intervention. Am J Surg. 2005;190(3):467-473.

16. Kal HB, Struikmans H. Radiotherapy during pregnancy: fact and fiction. Lancet Oncol. 20056(5):328-33.

17. Gentilini O, Cremonesi M, Toesca A, et al. Sentinel lymph node biopsy in pregnant patients with breast cancer. Eur J Nucl Med Mol Imaging. 2010;37(1):78-83.

18. Keleher A, Wendt R, Delpassand E, et al. The safety of lymphatic mapping in pregnant breast cancer patients using Tc-99m sulfur colloid. Breast J 2004. 2004;10(6):492-495.

19. Gentilini O, Cremonesi M, Trifi ro G, et al. Safety of sentinel node biopsy in pregnant patients with breast cancer. Ann Oncol. 2004;15(19):13481351 .

20. Dominici LS, Kuerer HM, Babiera G, et al. Wound complications from surgery in pregnancy-associated breast cancer (PABC). Breast Dis 2010. 2010;31(1):1-5.

21. Cardonick E, Iacobucci A. Use of chemotherapy during human pregnancy. Lancet Oncol. 2004;5(5):283-291.

22. Kal HB, Struikmans H. Radiotherapy during pregnancy: fact and fiction. Lancet Oncol. 2005;6(5):328-233.

23. Reed W, Hannisdal E, Skovlund E, et al. Pregnancy and breast cancer: a population-based study. Virchows Arch. 2003;443(1):44-50.

24. Aviles A, Neri N. Hematological malignancies and pregnancy: a final report of 84 children who received chemotherapy in utero. Clin Lymphoma. 2001;2(3):173-177.

25. Amant F, Loibl S, Neven P, et al. Breast cancer in pregnancy. Lancet. 2012;379(9815):570-579

26. Peccatori FA, Azim HA Jr, Scarfone G, et al. Weekly epirubicin in the treatment of gestational breast cancer (GBC). Breast Cancer Res Treat. 2009;115:591-594.

27. Amant F, Neven P, Van Calsteren K, et al. Treatment of cancer during pregnancy: the need for tailored strategies. J Clin Oncol. 2010;28:e304.

28. Bonilla L, Ben-Aharon I, Vidal L, et al. Dose-dense chemotherapy in nonmetastatic breast cancer: a systematic review and metaanalysis of randomized controlled trials. J Natl Cancer Inst 2010 2010;102(24):1845-1854. 
29. Amant F, Van Calsteren K, Halaska MJ. Long term cognitive and cardiac outcome after prenatal exposure to chemotherapy in children 18 months or older: Observational study. Lancet Oncol. 2012;13(3):256-264

30. Press MF, Cordon-Cardo C, Slamon DJ. Expression of the HER-2/neu proto-oncogene in normal human adult and fetal tissues. Oncogene. 1990;5(7):953-962.

31. Azim HA Jr, Azim H, Peccatori FA. Treatment of cancer during pregnancy with monoclonal antibodies: a real challenge. Expert Rev Clin Immunol. 2010;6(6):821-826.

32. Pentsuk N, van der Laan JW. An interspecies comparison of placental antibody transfer: new insights into developmental toxicity testing of monoclonal antibodies. Birth Defects Res B Dev Reprod Toxicol. 2009; $86: 328-344$

33. Pavlidis N, Pentheroudakis G. The pregnant mother with breast cancer: diagnostic and therapeutic management. Cancer Treat Rev. 2005;31(6):439-447.

34. Petrek JA, Dukoff R, Rogatko A. Prognosis of pregnancy-associated breast cancer. Cancer. 1991;67(4):869-872.

35. Sorosky JI, Sood AK, Buekers TE. The use of chemotherapeutic agents during pregnancy. Obstet Gynecol Clin North Am. 1997;24:591-599. 